\title{
Effects of Intravascular Laser Irradiation of Blood on Cognitive Function in a Stroke Survivor with Hyperhomocysteinemia: Dual Recuperations in Thalamus and Serum Homocysteine
}

\author{
Jia Yu Chang ${ }^{1}$, Chuan Ching Liu², I Ting Liu², Shin Tsu Chang*2,3,4 \\ ${ }^{1}$ Department of Medical Education, Taichung Veterans General Hospital, Taiwan \\ ${ }^{2}$ Department of Physical Medicine and Rehabilitation, Taichung Veterans General Hospital, Taichung, Taiwan \\ ${ }^{3}$ Department of Physical Medicine and Rehabilitation, Tri-Service General Hospital, School of Medicine, National Defense Medical Center, \\ Taipei, Taiwan \\ ${ }^{4}$ School of Medicine, Chung Shan Medical University, Taichung, Taiwan \\ *Corresponding author: Shin Tsu Chang, Department of Physical Medicine and Rehabilitation, Tri-Service General Hospital, School \\ of Medicine, National Defense Medical Center, Taipei; Department of Rehabilitation, Taichung Veterans General Hospital, Taichung; \\ School of Medicine, Chung Shan Medical University, Taichung, Taiwan
}

\section{ARTICLE INFO}

Received: 慧 March 15, 2019

Published: March 20, 2019

Citation: Jia Yu Chang, Chuan Ching Liu, I Ting Liu, Shin Tsu Chang. Effects of Intravascular Laser Irradiation of Blood on Cognitive Function in a Stroke Survivor with Hyperhomocysteinemia: Dual Recuperations in Thalamus and Serum Homocysteine. Biomed J Sci \& Tech Res 16(2)-2019. BJSTR. MS.ID.002817.

\section{ABSTRACT}

Keywords: Cognitive Impairment; Laser Phototherapy; Papez Circuit; Single Photon Emission Computed Tomography; Hyperhomocysteinemia

Abbreviations: HHcy: Hyperhomocysteinemia; ILIB: Intravenous Laser Irradiation of Blood; MMSE: Mini-Mental State Examination; SPECT: Single Photon Emission Computed Tomography

\section{Introduction}

Stroke is a major public health issue worldwide because of the high risk of unavoidable disability leading to a noteworthy burden of the patients themselves, the families and the society [1]. Cognitive impairment accounts for an important element of disability after stroke. Previous studies have shown a high prevalence of cognitive impairment in adults with stroke [2]. However, the pathophysiology of cognitive impairment has not been clarified yet. Hyperhomocysteinemia (HHcy) has been recognized as a risk factor for cardiovascular disease, stroke, vascular contributions to cognitive impairment and dementia, and Alzheimer's disease [3]. Nonetheless, the published methods for reduction of serum homocysteine did not have promising results yet. Intravenous laser irradiation of blood (ILIB) is a mini-invasive approach to provide phototherapy for adults with stroke [4]. Whether cognitive impairment may recuperate as a consequence of ILIB intervention has not been determined. We report herein cognitive impairment in a stroke survivor with HHcy, and after ILIB, remarkable regression of initial cognitive impairment occurred together with reduction of serum homocysteine. 


\section{Case Presentation}

A 50-year-old male who has history of hypertension without regular medications control suffered from difficulty in getting up and falling down on ground on 2015/07/26. At that time, his consciousness was clear, and right-sided limb/body was weak. He spoke haltingly. The first brain CT performed in a local hospital showed left intracranial hemorrhage and pontine infarction, and the second brain CT showed left thalamus intracerebral hemorrhage and intraventribular hemorrhage in a medical center. Right EVD insertion for monitor and CSF drainage were done on 2015/07/27. Since then, he had lots of inpatient rehabilitation in the past 2-3 years. Pain of the right shoulder took place in 2016, which made him visit our OPD for help until 2017/07/04. During outpatient visit, he showed right side limbs weakness with strength grading: upper limbs (right / left) 3/5; strength grading: lower limbs (right /left) 3/5. He had impaired walking ability and partially dependent activity of daily living, as well as right shoulder pain.
Cognitive impairment was examined by using Mini-Mental State Examination (MMSE) and recorded as 21 (Figure 1). The homocysteine level checked on 2017/08/11 showed value bigger than 50 umol/L. The brain perfusion scan with single photon emission computed tomography (SPECT) revealed decreased radioactivity over the left temporal, left parietal, left frontal, left striatum (esp. the putamen), left thalamus and right cerebellum, (Figure 2A). In the following 16 months, he underwent six-course ILIB, which method of approach has been previously detailed [4]. He stated that, after ILIB, he had no more shoulder pain, and had improved spirit and better sleeping quality, even spasticity remained. MMSE was recorded as 28 (Figure 1). The serum level of homocysteine reduced to $20.60 \mathrm{umol} / \mathrm{L}$ on $2018 / 12 / 01$. The second SPECT showed improvement in right cerebellum, both inferior frontal lobes, and left basal ganglion, as well as anterior thalamus (Figure 2B).

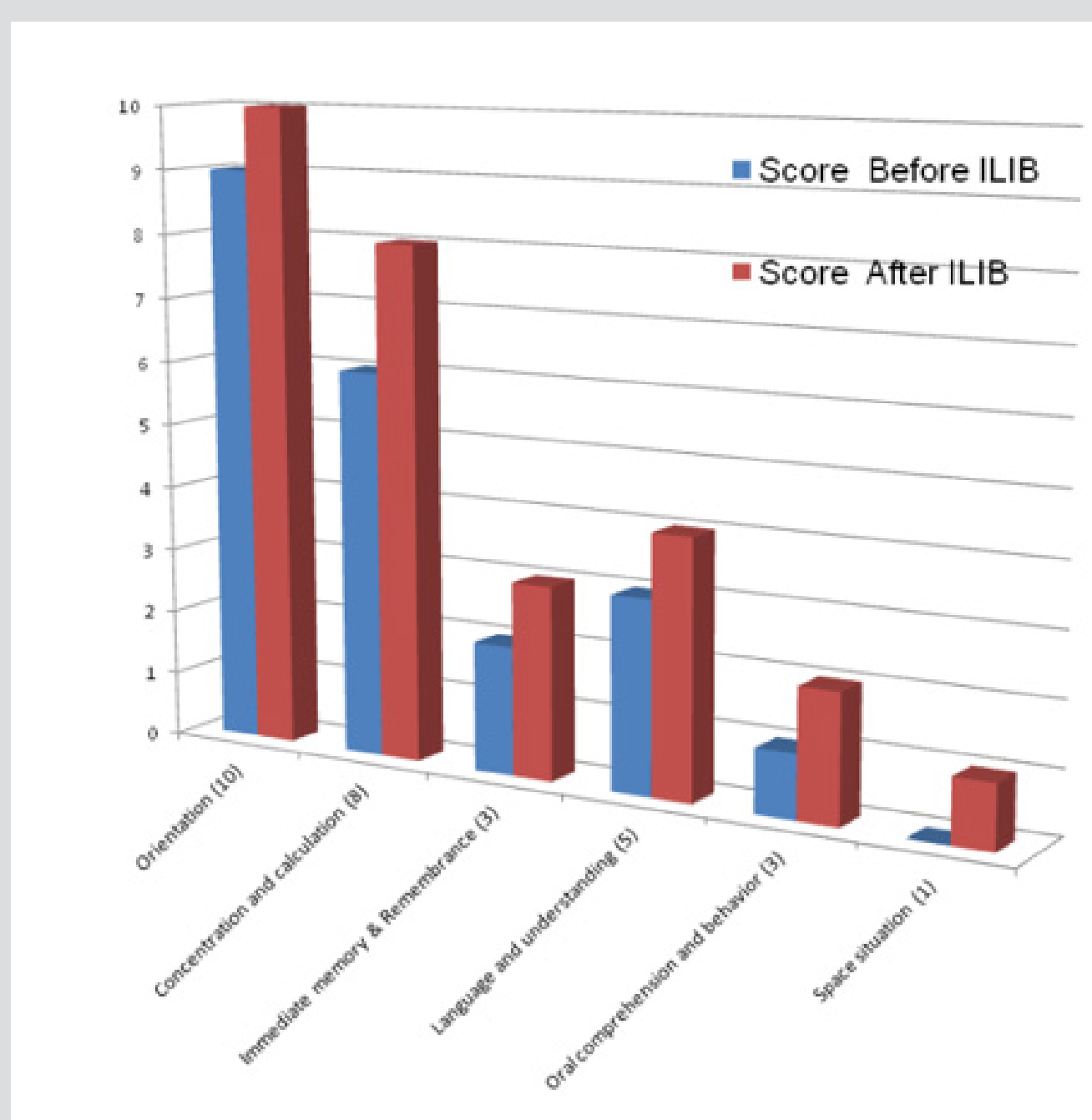

Figure 1: Mini-Mental State Examination (MMSE) of the patient, 21 before ILIB and 28 after ILIB. ILIB: intravenous laser irradiation of blood. 


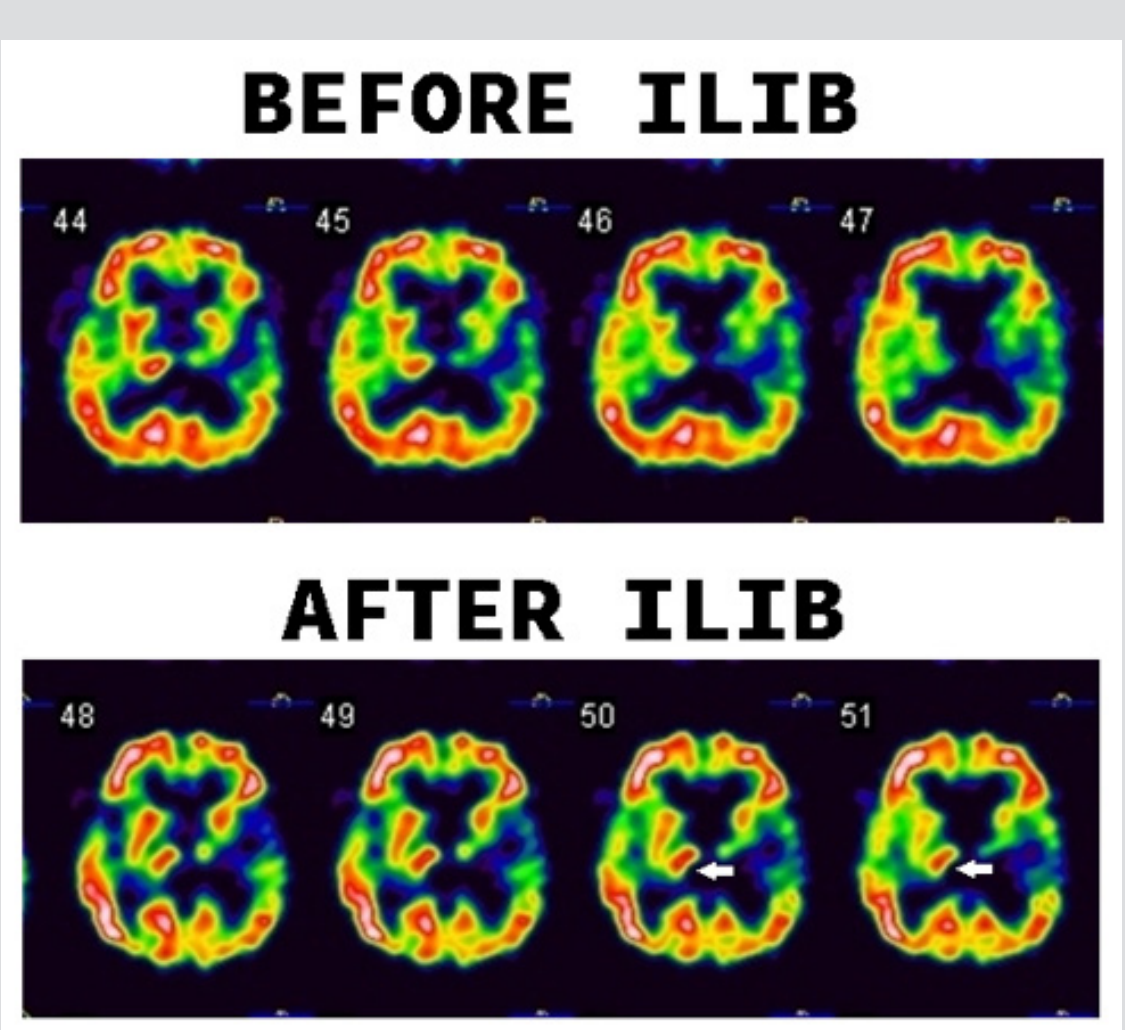

Figure 2: Tc-99m ethyl cysteinate dimer (ECD) brain perfusion SPECT study. Before (A) and after (B) the intervention of ILIB, there was increased uptake in the right thalamus (white arrow). ILIB: intravenous laser irradiation of blood.

\section{Discussion}

ILIB has been applied to many diseases, for instance, arthritis [57], fibromyalgia [8], hepatic disease [9], postpartum mastitis [10], diabetes mellitus [11-13], some systemic dys-metabolism [14,15], and regional cerebral hypoperfusion $[4,16]$, as its properties of improving oxygen supply and hence amplifying microcirculation. Cognitive impairment is common among patients after stroke episode. Several stroke characteristics are thought to be associated with an increased risk of cognitive impairment. Those heterogeneous risk factors have been reported as haemorrhagic stroke [17], lobar location of a parenchymal haemorrhage [18], occurrence of aphasia, multiple strokes [2], demographic characteristics (e.g., female sex, lower education, and racial background), or presence of known vascular risk factors [2]. $\mathrm{n}$ a study that recruited patients with different subtypes of strokes, cognitive function as indicated the MMSE was significantly impaired 6 months following the initial insult, and MMSE performance in post-stroke patients, $87 \%$ as ischemic of them, was significantly worse than that of the nonstroke control group [19]. A recent study showed, in "first-ever" ischemic stroke patients, the 30-year risk of cognitive impairment is 1.72 times that of the population; however, the risk is even greater for haemorrhagic stroke survivors [20].

Both global cerebral atrophy and medial temporal lobe atrophy are the most consistent predictors of cognitive impairment after stroke [21]. Our case had both ischemic and haemorrhagic episodes and possessed the high risk of cognitive impairment. Post-stroke cognitive impairment is considerably correlated with an increased mortality rate in ischemic stroke survivors [22,23]. As one of the clinical methods for cognitive assessment, SPECT plays an important role in functional demonstration. Regional CBF was examined with SPECT to reflect neural activity in individual brain region and to provide speculations on functional connectivity among distant areas [24]. Brain perfusion SPECT is a sensitive tool to assess the functional deficit in the early stages of cognitive impairment and is thus helpful in examining the pathophysiology of cognitive impairment in many diseases, such as dementia, Parkinson disease, cirrhotic liver, COPD, head injury, HIV, epilepsy. Until 1998, however, there was no article to assess stroke-related cognitive impairment by using brain SPECT before 1998. Hoffmann \& Watts (1998) firstly studied SPECT in five patients with first-ever, isolated brainstem stroke, and fouFnd that even brainstem stroke may result in substantial cognitive impairment and concluded that SPECT scanning is more sensitive than structural neuroimaging techniques. Egge [25] secondly tested stroke survivor with aneurismal subarachnoid hemorrhage by using SPECT in 2005 [26].

Researchers have confirmed both the Papez circuit and prefrontal-subcortical circuit as important neural structures for integration of cognitive function. The Papez circuit is involved in spatial learning and episodic memory, which circuit consists of the hippocampus, Para hippocampal gyrus, entorhinal cortex, mammillary bodies, and cingulate gyrus, and is interconnected by white matter 
tracts consisting of the fornix, mammillothalamic tract, cingulum bundle, and anterior thalamic nuclei [27]. In addition, three prefrontal-subcortical circuits are involved in Behavioral and cognitive alterations, e.g. the dorsolateral prefrontal circuits, the orbitofrontal circuits and the anterior cingulate circuits [28]. An ischemic stroke lesion related to post-stroke cognitive impairment often perturb the prefrontal-subcortical circuits including the bundles of white matter (corona radiate or centrum semiovale) [29], leading to executive deficits associated with prefrontal hypoperfusion [30]. Since disturbances in both local and global networks in the brain have been associated with deficits in cognitive function, therefore, disrupted or lately repaired neural network activity may provide valuable observation of a therapeutic intervention.

Interestingly, our case showed remarkable increase perfusion in the thalamus (Figure 2), indicating recovery of brain parenchymal lesions related to post-stroke cognitive impairment was attributed by the Papez circuit, rather than prefrontal-subcortical circuits. This finding suggests cognitive dysfunction in adult with stroke may be potentially reversible with intervention of ILIB. The reason for preference in Papez circuit was unknown and should be investigated later. Increasing evidence had confirmed that homocysteine is associated with distinct category of cardiovascular and cerebrovascular diseases [31,32]. HHcy is related with functional disability in the any phase of stroke, and many articles confirmed a significant correlation between lower cognitive function and raised homocysteine level [33-35]. It has been shown that patients with acute stroke with elevated serum homocysteine levels are at an increased risk for early neurological deterioration [36]. Increased serum levels of Hcy may cause neurotoxicity and inflammatory activity. Direct toxicity and vascular endothelial injury in ischemic stroke patients whose inflammatory system reacts most extremely may be at bigger risk for cerebrovascular diseases [37].

With respect to the treatment, HHcy represents a potentially modifiable risk factor for stroke, but the published methods for reduction did not have promising results. [38] Our ILIB provides phototherapy. It has been known that some proteins or enzymes are photo-sensitive and can be activated to exert specific function, for instance, vascular endothelial growth factor, cyclooxygenase- 2 and matrix metalloproteases, as well as photosensitive ganglion cell in eye retina [39]. Nitrile hydratase can also catalyse hydration of nitriles to the corresponding amides when unbinding a nitric oxide at light at the non-heme iron center, but inactive if binding with nitric oxide at dark [40]. Homocysteine can be metabolized by two pathways: demethylation by methionine synthase and transculturation by cystathionine beta-synthase [32]. It was interested to observe if both enzymes, or either one, would be activated after exposure of ILIB, which hence to reduce serum level of homocysteine. Further study will be elucidated. Our case showed a remarkable improvement of cognitive impairment correlated with cognitive testing after six-course ILIB, which improved the perfusion in thalamus and decreased the serum level of homocysteine. Further studies on a larger number of patients are needed to test this hypothesis.

\section{Conclusion}

The fact of the recuperation in cerebral perfusion of thalamus and serum level of homocysteine in the present case after intervention of ILIB suggest that ILIB might possess dual effect to increase thalamus perfusion and, simultaneously, enhancing catabolism of homocysteine.

\section{References}

1. Feigin VL, Forouzanfar MH, Krishnamurthi R, Mensah GA, Connor M, et al. (2014) Global Burden of Diseases, Injuries, and Risk Factors Study 2010 (GBD 2010) and the GBD Stroke Experts Group. Global and regional burden of stroke during 1990-2010: findings from the Global Burden of Disease Study 2010. Lancet 383(9913): 245-254.

2. Pendlebury ST, Rothwell PM (2009) Prevalence, incidence, and factors associated with pre-stroke and post-stroke dementia: a systematic review and meta-analysis. Lancet Neurol 8(11): 1006-1018.

3. Beydoun MA, Beydoun HA, Gamaldo AA, Teel A, Zonderman AB, et al. (2014) Epidemiologic studies of modifiable factors associated with cognition and dementia: systematic review and meta-analysis. BMC Public Health 14: 643.

4. Yang WH, Lin SP, Chang ST (2017) Case report: Rapid improvement of crossed cerebellar diaschisis after intravascular laser irradiation of blood in a case of stroke. Medicine (Baltimore) 96(2): e5646.

5. Timofeyev VT, Poryadin GV, Goloviznin MV (2001) Laser irradiation as a potential pathogenetic method for immunocorrection in rheumatoid arthritis. Pathophysiology 8(1): 35-40.

6. Chiran DA, Litscher G, Weber M, Ailioaie LM, Ailioaie C, et al. (2013) Intravenous laser blood irradiation increases efficacy of etanercept in selected subtypes of juvenile idiopathic arthritis: an innovative clinical research approach. Evid Based Complement Alternat Med, pp. 168134.

7. Chiran DA, Weber M, Ailioaie LM, Moraru E, Ailioaie C, et al. (2014) Intravenous laser blood irradiation and tocilizumab in a patient with juvenile arthritis. Case Rep Med 2014: 923496.

8. Momenzadeh S, Abbasi M, Ebadifar A, Aryani M, Bayrami J, et al. (2015) The intravenous laser blood irradiation in chronic pain and fibromyalgia. J Lasers Med Sci 6(1): 6-9.

9. Babaev AV, Gogolev DE, Reiner OV, Korochkin IM, Fandeev AV, et al. (2012) Effect of intravenous low-intensity laser irradiation of the blood on clinical and laboratory parameters of hepatocellular insufficiency. Bull Exp Biol Med 153(5): 754-757.

10. Mokmeli S, Khazemikho N, Niromanesh S, Vatankhah Z (2009) The application of low-level laser therapy after cesarean section does not compromise bloodprolactin levels and lactation status. Photomed Laser Surg 27(3): 509-512.

11. Kazemi Khoo N, Iravani A, Arjmand M, Vahabi F, Lajevardi M, et al. (2013) A metabolomic study on the effect of intravascular laser blood irradiation on type 2 diabetic patients. Lasers Med Sci 28(6): 1527-1532.

12. KazemiKhoo N, Ansari F (2015) Blue or red: which intravascular laser light has more effects in diabetic patients? Lasers Med Sci 30(1): 363366.

13. Kazemi Khoo N, Sarafnejad AF, Ansari F, Mehdipour P (2016) Modifying effect of intravenous laser therapy on the protein expression of arginase and epidermal growth factor receptor in type 2 diabetic patients. Lasers Med Sci 31(8): 1537-1545. 
14. Huang SF, Tsai YA, Wu SB, Wei YH, Tsai PY, et al. (2012) Effects of intravascular laser irradiation of blood in mitochondria dysfunction and oxidative stress in adults with chronic spinal cord injury. Photomed Laser Surg 30(10): 579-586.

15. Mikhaylov VA (2015) The use of Intravenous Laser Blood Irradiation (ILBI) at 630-640 nm to prevent vascular diseases and to increase life expectancy. Laser Ther 24(1): 15-26.

16. Liu EY, Chang ST (2019) Benefits of intravascular laser irradiation of blood on motor and sensory recovery viewing from brain function images: portrait of a case with chronic Sjögren's syndrome, transverse myelitis, and Guillain-Barré syndrome. Biomedical Journal of Scientific and Technical Research 14(4): 2109

17. Corraini P, Henderson VW, Ording AG, Pedersen L, Horvath-Puho E, et al. (2017) Long-term risk of dementia among survivors of ischemic or hemorrhagic stroke. Stroke 48(1): 180-186.

18. Moulin S, Labreuche J, Bombois S, Rossi C, Boulouis G, et al. (2016) Dementia risk after spontaneous intracerebral haemorrhage: a prospective cohort study. Lancet Neurol 15(8): 820-829.

19. Kase CS, Wolf PA, Kelly Hayes M, Kannel WB, Beiser A, et al. (1988) Intellectual decline after stroke: The Framingham Study. Stroke 29(4): 805-812.

20. Corraini P, Henderson VW, Ording AG, Pedersen L, Horvath Puho E, et al. (2017) Long-term risk of dementia among survivors of ischemic or hemorrhagic stroke. Stroke 48(1): 180-186.

21. Casolla B, Caparros F, Cordonnier C, Bombois S, Hénon H, et al. (2018) Biological and imaging predictors of cognitive impairment after stroke: a systematic review. J Neurol.

22. Desmond DW, Moroney JT, Sano M, Stern Y (2002) Mortality in patients with dementia after ischemic stroke. Neurology 59(4): 537-543.

23. Gao CY, Lian Y, Zhang M, Zhang LL, Fang CQ, et al. (2016) Association of dementia with death after ischemic stroke: A two-year prospective study. Exp Ther Med 12(3): 1765-1769.

24. K Akanuma, K Meguro, Y Kato, Y Takahashi, K Nakamura, et al. (2016) Impaired attention function based on the Montréal Cognitive Assessment in vascular dementia patients with frontal hypoperfusion: the Osaki-Tajiri project. J. Clin. Neurosci 28: 128-132.

25. Hoffmann M, Watts A (1988) Cognitive dysfunction in isolated brainstem stroke: a neuropsychological and SPECT study. J Stroke Cerebrovasc Dis $7(1):$ 24-31.

26. Egge A, Waterloo K, Sjøholm H, Ingebrigtsen T, Forsdahl S, et al. (2005) Outcome 1 year after aneurysmal subarachnoid hemorrhage: relation between cognitive performance and neuroimaging. Acta Neurol Scand 112(2): 76-80.

27. Vann SD, Nelson AJ (2015) The mammillary bodies and memory: More than a hippocampal relay. Prog Brain Res 219: 163-185.

\section{ISSN: 2574-1241}

\section{DOI: 10.26717/BJSTR.2019.16.002817}

Shin Tsu Chang. Biomed J Sci \& Tech Res

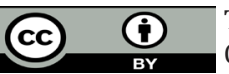

This work is licensed under Creative

Commons Attribution 4.0 License

Submission Link: https://biomedres.us/submit-manuscript.php
28. Mega MS, Cummings JL (1994) Frontal subcortical circuits and neuropsychiatric disorders. J Neuropsychiatry Clin Neurosci 6(4): 358370 .

29. Vataja R, Pohjasvaara T, Mäntylä R, Ylikoski R, Leppävuori A, et al. (2003) MRI correlates of executive dysfunction in patients with ischaemic stroke. Eur J Neurol 10(6): 625-631.

30. Lanna ME, Alves CE, Sudo FK, Alves G, Valente L, et al. (2012) Cognitive disconnective syndrome by single strategic strokes in vascular dementia. J Neurol Sci 322(1-2): 176-183.

31. Khan U, Crossley C, Kalra L, Rudd A, Wolfe CD, et al. (2008) Homocysteine and its relationship to stroke subtypes in a UK black population: The South London Ethnicity and Stroke Study. Stroke 39(11): 2943-2949.

32. Ganguly P, Alam SF (2015) Role of homocysteine in the development of cardiovascular disease. Nutr J 14: 6.

33. Blasko I, Jellinger K, Kemmler G, Krampla W, Jungwirth S, et al. (2008) Conversion from cognitive health to mild cognitive impairment and AD: Prediction by plasma amyloid beta 42 , medial temporal lobe atrophy and homocysteine. Neurobiol. Aging 2008, 29(1): 1-11.

34. Moustafa AA, Hewedi DH, Eissa AM, Myers CE, Sadek HA (2012) The relationship between associative learning, transfer generalization, and homocysteine levels in mild cognitive impairment. PLoS ONE 7(9): e46496.

35. Faux NG, Ellis KA, Porter L, Fowler CI, Laws SM, et al. (2011) Homocysteine, vitamin B12 and folic acid levels in AD, mild cognitive impairment, and healthy elderly: Baseline characteristics in subjects of the Australian Imaging biomarker Lifestyle Study. J Alzheimer's Dis 27(4): 909-922.

36. Kwon HM, Lee YS, Bae HJ, Kang DW (2014) Homocysteine as a Predictor of Early Neurological Deterioration in Acute Ischemic Stroke. Stroke 45(3): 871-873.

37. Moretti R, Caruso P (2019) The Controversial Role of Homocysteine in Neurology: From Labs to Clinical Practice. Int J Mol Sci 20(1): 231.

38. Price BR, Wilcock DM, Weekman EM (2018) Hyperhomocysteinemia as a risk factor for vascular contributions to cognitive impairment and dementia. Front Aging Neurosci 10: 350.

39. Bhuvaneswari R, Gan YY, Soo KC, Olivo M (2009) The effect of photodynamic therapy on tumor angiogenesis. Cell Mol Life Sci 66(14): 2275-2283.

40. Noguchi T, Nojiri M, Takei K, Odaka M, Kamiya N (2003) Protonation structures of Cys-sulfinic and Cys-sulfenic acids in the photosensitive nitrile hydratase revealed by Fourier transform infrared spectroscopy. Biochemistry 42(40): 11642-11650.

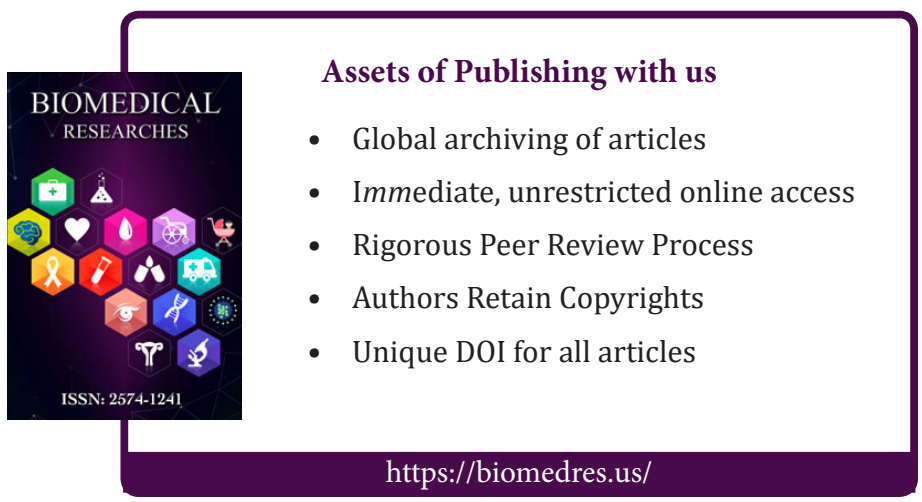

